\title{
Upper limits on resonance contributions to proton-proton elastic scattering in the c.m. mass range $2.05-2.85 \mathrm{GeV} / \mathrm{c}^{2}$
}

\author{
H. Rohdjeß ${ }^{1}$, M. Altmeier ${ }^{1}$, F. Bauer ${ }^{2}$, J. Bisplinghoff ${ }^{1}$, R. Bollmann ${ }^{2}$, K. Büßer ${ }^{2}$, M. Busch ${ }^{1}$, O. Diehl ${ }^{1}$, F. Dohrmann ${ }^{2}$, \\ H.P. Engelhardt ${ }^{1}$, J. Ernst ${ }^{1}$, P.D. Eversheim ${ }^{1}$, K.O. Eyser ${ }^{2}$, O. Felden ${ }^{3}$, R. Gebel ${ }^{3}$, A. Groß ${ }^{2}$, R. Groß-Hardt ${ }^{1}$, F. Hinterberger ${ }^{1}$, \\ R. Langkau ${ }^{2}$, J. Lindlein ${ }^{2}$, R. Maier ${ }^{3}$, F. Mosel $^{1}$, D. Prasuhn ${ }^{3}$, P. von Rossen ${ }^{3}$, N. Scheid ${ }^{1}$, M. Schulz-Rojahn ${ }^{1}$, F. Schwandt ${ }^{1}$, \\ V. Schwarz ${ }^{1}$, W. Scobel ${ }^{2}$, H.-J. Trelle ${ }^{1}$, K. Ulbrich ${ }^{1}$, E. Weise ${ }^{1}$, A. Wellinghausen ${ }^{2}$, K. Woller ${ }^{2}$, and R. Ziegler ${ }^{1}$ \\ 1 Helmholtz-Institut für Strahlen- und Kernphysik, Universität Bonn, D-53115 Bonn, Germany \\ 2 Institut für Experimentalphysik, Universität Hamburg, D-22761 Hamburg, Germany \\ ${ }^{3}$ Institut für Kernphysik, Forschungszentrum Jülich, D-52425 Jülich, Germany
}

Received: date / Revised version: date

\begin{abstract}
Recently published excitation functions in proton-proton $(p p)$ elastic scattering observables in the laboratory energy range $0.5-2.5 \mathrm{GeV}$ provide an excellent data base to establish firm upper limits on the elasticities $\eta_{\mathrm{el}}=\Gamma_{\mathrm{el}} / \Gamma_{\text {tot }}$ of possible isovector resonant contributions to the Nucleon-Nucleon (NN) system. Such contributions have been predicted to arise from dibaryonic states, with c.m. masses between $2.1-2.9 \mathrm{GeV} / c^{2}$, but have not been confirmed experimentally. A method to determine quantitatively the maximum value of $\eta_{\mathrm{el}}$ compatible with experimental data is presented. We use energy dependent phase shift fits to the $p p$-data base to model the non-resonant interaction. Based upon the differential cross-section data measured by the EDDA Collaboration an unbiased statistical test is constructed to obtain upper limits on $\eta_{\mathrm{el}}$, that exclude larger values with a $99 \%$ confidence level. Results in the c.m. mass range $2.05-2.85 \mathrm{GeV} / c^{2}$ and total widths of $10-100 \mathrm{MeV} / c^{2}$ in the partial waves ${ }^{1} S_{0},{ }^{1} D_{2},{ }^{3} P_{0},{ }^{3} P_{1}$, and ${ }^{3} F_{3}$ are presented and discussed.
\end{abstract}

PACS. 25.40.Cm Elastic proton scattering - 13.75.Cs Nucleon-nucleon interactions - 14.20.Pt Dibaryons - 11.80.Et Partial-wave analysis

\section{Introduction}

Practically all established hadronic states can be explained at the quark-level as bound states of either three quarks (baryons) or quark-antiquark states (mesons). With the advent of QuantumChromo-Dynamics (QCD), the by now accepted theory of the strong interaction, a new degree of freedom - color - was introduced. The non-observation of for instance single quarks or four quark states can be explained, when color-confi nement is assumed, restricting the color-fi eld to small volumes, such that only color-singlet states can be observed. However, within the framework of QCD, other color-singlet bound states, made up by certain other number of quarks and anti-quarks or by gluons, are not ruled out. Unfortunately, due to the non-abelian nature of QCD the coupling between quarks in the low energy regime is strong, making a rigorous calculation of low-energy phenomena, such as bound state properties, within QCD impossible. However, the progress of lattice QCD may provide us with a way to calculate numerically the mass and other properties of such objects.

Send offprint requests to: F. Hinterberger, HISKP, Universität Bonn, Nussallee 14-16, D-53115 Bonn, Germany

Correspondence to: fh@ hiskp.uni-bonn.de
In the low and intermediate energy regime phenomenological and QCD-inspired effective theories have been developed, e.g. the Bag-model, the Skyrme model and the ConstituentQuark model, which have been reasonably successful to describe the mass-spectrum of most of the known mesons $(q \bar{q})$ and baryons $(q q q)$. The possibility to form other color singlet states, such as dibaryons ( $q q q q q q)$, baryonium $(q q \bar{q} \bar{q})$, pentaquarks $(q q q q \bar{q})$, hybrids $(q \bar{q} g)$ and glueballs $(g g, g g g)$ was soon recognized and sparked intense theoretical and experimental activities.

Jaffe [1] predicted a bound dibaryonic state made from two up $(u)$, down $(d)$ and strange $(s)$ quarks each, using the MIT bag model. This H-particle with a calculated mass below the $\Lambda \Lambda$-threshold was expected to decay weakly with a narrow width. These calculations were extended to the non-strange quark sector [2-4]. Here, relative momenta of two colored cluster were introduced, such that the angular momentum barrier hinders the decay, which requires the rearrangement in two colorneutral three-quark clusters [5, 6]. Like for baryons, a whole spectrum of dibaryonic states was predicted. However, only the lowest lying states were hoped to have a decay width small enough to be observed experimentally, although all states were predicted to be above the inelastic $(N N \pi)$ threshold. 
The mass spectrum of dibaryonic states have also been obtained in a number of phenomenological or QCD-inspired models, e.g. the constituent-quark [7, 8], diquark-cluster [9] and the Skyrme model $[10,11]$. The prediction of the masses scattered considerably, but clustered in the 2.1-2.4 GeV range for the lowest states. All model parameters must be fi xed by the properties of the known mesons and baryons. Since it is questionable if these parameters are unchanged in a six-quark system, the theoretical uncertainties of all predictions are large. Another problem is, that most dibaryonic states have open decay channels $[12,13]$. The coupling of the decay-channel to the six-quark system may alter the energy and width of the states considerably.

Calculations within the Cloudy-Bag Model where the internal quark wave functions in the bag are coupled at the bag radius to external $N \Delta, N N^{*}$ and $\Delta \Delta$ channels, modeled by meson-exchange potentials, have led to predictions [14-19] of the lowest dibaryonic states at higher energies of about 2.7 $2.8 \mathrm{GeV}$.

In the 1980s several experiments were devoted to search for evidence of dibaryonic states (see Refs. [20-26] for a review). Despite a large effort none of the numerous positive claims survived experimental verifi cation. Especially, precise excitation functions of the differential cross-section in the kinetic energy range $500-1200 \mathrm{MeV}$ [27] and the analyzing power in the kinetic energy range 655 - $1017 \mathrm{MeV}$ [28] measured at SATURNE II by Garçon et al. did not show any evidence for narrow resonances. Candidates reported in Refs. [29, 30] and analyzed in [31] were not verifi ed experimentally by Beurtey et al. [32]. Also candidates reported in Refs. [33, 34] were not verifi ed in later remeasurements [35]. The same holds true for candidates reported in Refs. [36, 37]. The candidates reported in Refs. [38-40] have not yet been confi rmed by other measurements.

Precise and internally consistent excitation functions of the elastic proton-proton scattering, i.e. differential cross-sections $[41,42]$, analyzing powers $[43,44]$ and spin correlation parameters $[45,46]$, have recently been measured by the EDDA Collaboration in the kinetic energy range 0.45 to $2.5 \mathrm{GeV}$. There is no evidence for narrow resonances in those data. The aim of the present paper is to deduce upper limits on resonance contributions to proton-proton elastic scattering in the c.m. mass range 2.05-2.85 GeV/c $\mathrm{c}^{2}$ using the data of the EDDA experiment.

\section{Dibaryons}

Loosely speaking, all states with baryon-number equal two can be termed dibaryon. However, in the context of this paper we will consider only states as dibaryons which cannot be explained trivially as a bound state or a resonance in a system of two baryons, like the deuteron. Good candidates would be resonances with widths considerably smaller than the typical width of baryon resonances, i.e. smaller than about $100 \mathrm{MeV}$.

The structures in spin-dependent total cross-sections $\Delta \sigma_{T}$ and $\Delta \sigma_{L}$ of $p p$ elastic scattering at the ZGS $[47,48]$ were soon interpreted as resonances in the ${ }^{1} D_{2}$ and ${ }^{3} F_{3}$ partial waves [4951]. Modern phase shift analysis show counter-clockwise rotation in the Argand-plot with widths of $80-150 \mathrm{MeV}$ at energies of $2170 \mathrm{MeV}(2250 \mathrm{MeV})$ for the ${ }^{1} D_{2}\left({ }^{3} F_{3}\right)$ partial waves

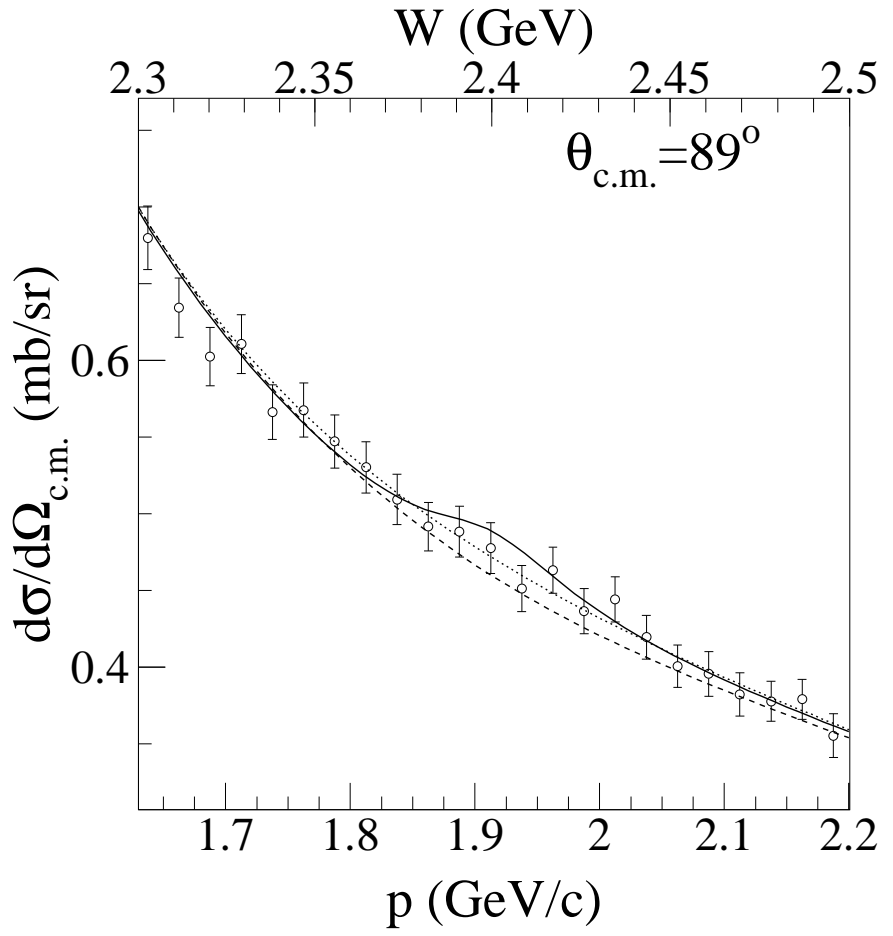

Fig. 1. Example of an excitation function from [42] in comparison to a best-fit PSA without (dotted) and with (solid line) a resonance in the ${ }^{1} S_{0}$ partial wave at $W_{\mathrm{R}}=2.4 \mathrm{GeV}$, with a total width of $50 \mathrm{MeV}$ and a partial elastic width of $2.8 \mathrm{MeV}$. The non-resonant part of the latter is shown as the dashed line. The presence of this resonance is excluded with $99 \% \mathrm{CL}$, based on 28 excitation functions at different scattering angles.

$[52,53]$. However, these structures can easily be understood by the opening of the $N \Delta$ inelastic channel [54], since they appear in the lowest $N \Delta$ partial waves at approximately the $N \Delta$ mass. This interpretation is also supported by coupled channel $[15,55,56]$ and Fadeev [57] calculations, so that these structures are widely accepted as dibaryons in the trivial sense.

Resonances with widths considerably below $100 \mathrm{MeV}$ would not be easily explained by conventional models, and would be good candidates for exotic dibaryonic states.

\section{Determination of Upper Limits of resonance contributions}

Recent measurements of high-precision excitation functions of the elastic proton-proton scattering cross-sections [41, 42], analyzing powers $[43,44]$ and spin correlation parameters $[45$, 46] in the energy range 0.45 to $2.5 \mathrm{GeV}$ kinetic energy have shown no evidence for energy-dependent structures within the experimental errors. This observation can be used to set upper limits quantitatively on the coupling of narrow resonances to the elastic channel in proton-proton elastic scattering. In this context we consider resonances to be "narrow", when the width is considerably smaller than that of the lowest excited baryons, i.e. total widths not larger than $100 \mathrm{MeV}$. The analysis we have performed attempts to provide an unbiased statis- 
tical test on the compatibility of resonance contributions with the data of the EDDA experiment at COSY [41-46]. At the beginning of this study the cross-section and analyzing power data were used [58]. But it turned out that the sensitivity of the analyzing power data was very low. Also, the EDDA data on excitation functions of the spin correlation parameters $A_{N N}$, $A_{S S}$ and $A_{S L}$ [46] were not used. This is due to the fact that those data have a rather low statistical accuracy when compared to the cross-section data. Therefore, the upper limits of the present study are based solely on the very precise excitation functions of the differential cross-sections [42]. The method we employed is described in the next sections. The upper limits for the elastic width of resonances in the partial waves ${ }^{1} S_{0}$, ${ }^{1} D_{2},{ }^{3} P_{0},{ }^{3} P_{1}$, and ${ }^{3} F_{3}$ with c.m. energies between 2.05 and $2.85 \mathrm{GeV}$ and total widths in the $10-100 \mathrm{MeV}$ range are presented in Sec. 4.

\subsection{Overview}

The aim of this paper is to construct an unbiased statistical test for the compatibility of an isolated resonance in a single partial wave with the experimental excitation functions. To achieve this, we need to distinguish the contributions of the resonance from that of non-resonant scattering. Unfortunately, to date no theory or even model exists which describes nucleonnucleon elastic scattering far above the inelastic threshold, i.e. $300 \mathrm{MeV}$, quantitatively. Only qualitative agreement has been reached within meson-exchange models for energies below 1.0 $\mathrm{GeV}$ [59]. Therefore, we rely on energy-dependent phase-shifts $[60,66]$ to account for the non-resonant scattering contribution, where the parameterization of the energy-dependence is chosen not to allow for rapid changes of partial-wave amplitudes as they arise from resonances. To this end, an energy-dependent phase shift analysis (PSA), tailored to give an optimal fi t to the test data set, serves as the null hypothesis. When we want to test the compatibility of an assumed resonant contribution in the partial wave ${ }^{2 S+1} L_{J}$ with given energy, width and partial elastic width, we add the resulting resonant amplitude to the corresponding partial wave amplitude. If we compared the result, i.e. the prediction of PSA plus the resonance, to the experimental data the test would be biased in the following sense: the non-resonant contribution was fi tted to the data under the assumption that no resonance contributes. This bias can be removed if we now refi $\mathrm{t}$ all phase-parameters with the resonance included in the appropriate partial-wave amplitude to obtain a modifi ed solution (PSA'). The result of this modifi ed PSA' together with the resonance is the best fit to the data, with the assumption that the resonance exists and its comparison with the test data set allows to test the resonance hypothesis.

In Fig. 1 an example is shown, where the dotted line represents the null-hypothesis (PSA), the solid line is the result for the resonance-hypothesis. The dashed line shows the contribution from the modifi ed PSA' representing the non-resonant scattering in the presence of the isolated resonance. The PSA' moves away from the data to best accommodate the resonance, whose parameters remain fi xed when the phase shifts of PSA' are adjusted. Note, that the fi $t$ will be performed on several excitation functions at different angles simultaneously. In Fig. 2
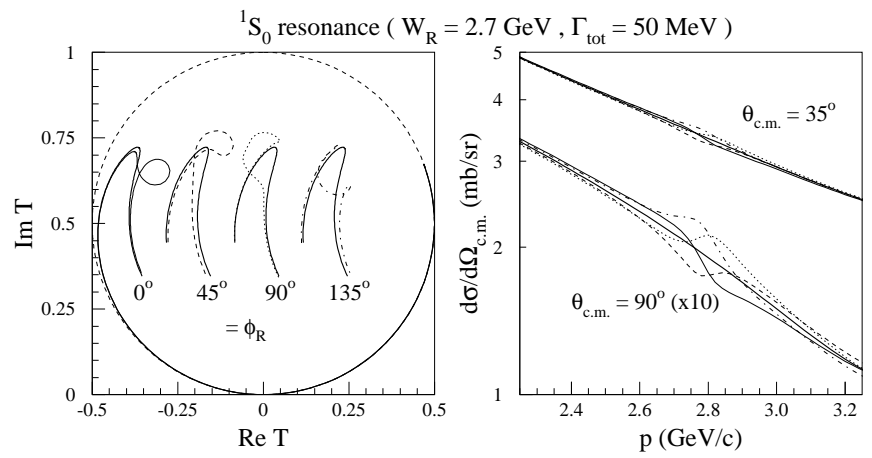

Fig. 2. Argand-diagram (left) and differential cross-sections (right), assuming a ${ }^{1} S_{0}$-resonance at $2.7 \mathrm{GeV}$ with a width of $50 \mathrm{MeV}$ for four different values of the phase $\phi_{R}$ (offset to the right for $\phi_{R} \neq 0$ ).

the effect of the relative phase $\phi_{\mathrm{R}}$ between resonant and nonresonant amplitudes is shown for a ${ }^{1} S_{0}$-resonance at $2.7 \mathrm{GeV}$ with a width of $50 \mathrm{MeV}$. The fi gure shows the Argand-diagram (left) and differential cross-sections (right) for four different values of the phase $\phi_{\mathrm{R}}$.

Finally, the comparison of the experimental data and the null- and resonance-hypothesis are subject to a statistical analysis. Based on a $\chi^{2}$-test, the confi dence level (CL) for excluding the resonant with the chosen parameters is determined.

The different steps of the analysis and the formalism employed are described in the following sections.

\subsection{Formalism}

Elastic scattering of two identical spin $1 / 2$ particles can be described by 5 complex amplitudes [61, 62], when basic invariance properties (parity, time-reversal, Pauli-principle) of the strong interaction are taken into account. A number of different choices for these amplitudes have appeared in the literature $[53,63,64]$. Here, we will use the amplitudes $H_{1}, \ldots, H_{5}$ introduced by Arndt, Roper, Bryan, Clark, VerWest and Signell [53].

These amplitudes can be expanded in partial waves ${ }^{2 S+1} L_{J}$, according to the relative orbital angular momentum $L$, total spin $S$ and total angular momentum $J=|L-S|, \ldots,|L+S|$ of the two-proton wave function. To satisfy the Pauli-principle the total wave function must be antisymmetric with respect to particle-interchange, so that only partial waves with $(-1)^{L+S+1}$ equal to -1 contribute to proton-proton elastic scattering. In Tab. 1 the lowest partial waves in NN scattering are listed.

Since total angular momentum and parity - given by $\pi=$ $(-1)^{L}-$ are conserved, only partial waves with the same $J^{\pi}$ can couple, a condition only satisfi ed for spin-triplet partial waves with $L=J \pm 1$. If we restrict ourselves to uncoupled partial waves ${ }^{2 S+1} L_{j}$ the corresponding $S$-matrix element $S_{j}$ is simply a complex number and can be parameterized by the corresponding phase-shift $\delta_{j}$ viz.

$$
S_{j}=e^{2 i \delta_{j}}
$$

Below the pion production threshold, unitarity requires $\left|S_{j}\right|=$ 1 and $\delta_{j}$ to be real, however at higher energies the open in- 
Table 1. Partial waves for $\mathrm{J}=0 \ldots 4$ in nucleon-nucleon scattering using the notation ${ }^{2 S+1} L_{J}$ for a given isospin $I$.

\begin{tabular}{|c|c|c|c|c|c|c|c|c|c|c|c|}
\hline \multirow[t]{2}{*}{$\overline{\bar{I}}$} & & \multicolumn{10}{|c|}{$\overline{J^{\pi}}$} \\
\hline & & $0^{+}$ & $0^{-}$ & $1^{+}$ & $1^{-}$ & $2^{+}$ & $2^{-}$ & $3^{+}$ & $3^{-}$ & $4^{+}$ & $4^{-}$ \\
\hline \multirow{3}{*}{0} & $J-1$ & - & - & ${ }^{3} S_{1}$ & - & - & - & ${ }^{3} D_{3}$ & - & - & - \\
\hline & $J$ & - & - & - & ${ }^{1} P_{1}$ & ${ }^{3} D_{2}$ & - & - & ${ }^{1} F_{3}$ & ${ }^{3} G_{4}$ & - \\
\hline & $J+1$ & - & - & ${ }^{3} D_{1}$ & - & - & - & ${ }^{3} G_{3}$ & - & - & - \\
\hline \multirow{3}{*}{1} & $J-1$ & - & - & - & - & - & ${ }^{3} P_{2}$ & - & - & - & ${ }^{3} F_{4}$ \\
\hline & $J$ & ${ }^{1} S_{0}$ & - & - & ${ }^{3} P_{1}$ & ${ }^{1} D_{2}$ & - & - & ${ }^{3} F_{3}$ & ${ }^{1} G_{4}$ & - \\
\hline & $J+1$ & - & ${ }^{3} P_{0}$ & - & - & - & ${ }^{3} F_{2}$ & - & - & - & ${ }^{3} H_{4}$ \\
\hline
\end{tabular}

elastic channels make $\left|S_{j}\right| \leq 1$ and thus phase-shifts become complex.

Lets assume an isolated resonance with angular momentum $j$, resonance energy $W_{\mathrm{R}}$ and total width $\Gamma_{\text {tot }}$ coupled to the NN elastic channel. When we use $S_{j, \mathrm{nr}}$ to describe the nonresonant scattering the $S$-matrix element is given by [65]

$$
S_{j}=S_{j, \mathrm{nr}}-i \frac{e^{2 i\left(\operatorname{Re} \delta_{j}+\phi_{\mathrm{R}}+\phi_{\mathrm{c}}\right)} \Gamma_{\mathrm{el}}}{W-W_{\mathrm{R}}+i \Gamma_{\mathrm{tot}} / 2} .
$$

where $W=\sqrt{s}$ is the total energy of the $p p$ c.m.-system, $\Gamma_{\text {el }}$ the partial width for the coupling of the resonance to the elastic channel, $\phi_{\mathrm{R}} \in[0, \pi[$ an unknown, arbitrary relative phase of the resonant and non-resonant amplitudes, and $\phi_{\mathrm{c}}$ the Coulomb-phase, since the phase-shifts $\delta_{j}$ are considered as bare nuclear phase-shifts with the Coulomb interaction taken out.

The corresponding $T$-matrix element is then given by

$$
T_{j}=\frac{S_{j}-1}{2 i}=T_{j, \mathrm{nr}}-\underbrace{\frac{1}{2} \cdot \frac{e^{2 i\left(\operatorname{Re} \delta_{j}+\phi_{\mathrm{R}}+\phi_{\mathrm{c}}\right)} \Gamma_{\mathrm{el}}}{W-W_{\mathrm{R}}+i \Gamma_{\mathrm{tot}} / 2}}_{T_{j, \mathrm{res}}} .
$$

Taking the partial wave expansion of the amplitudes $H_{1 \ldots 5}$ from Ref. [53] we obtain for a resonance in a spin-singlet partial wave a modifi ed amplitude $H_{1}$

$$
H_{1}=H_{1, \mathrm{nr}}-(2 j+1) T_{j, \mathrm{res}} P_{j}
$$

and for a resonance in an uncoupled triplet partial wave $H_{2}$ and $H_{3}$ are modifi ed

$$
\begin{aligned}
& H_{2}=H_{2, \mathrm{nr}}-\left\{(2 j+1) P_{j}-P_{j}^{1} \cot \theta_{\text {c.m. }}\right\} T_{j, \text { res }} \\
& H_{3}=H_{3, \mathrm{nr}}-\frac{P_{j}^{1}}{\sin \theta_{\text {c.m. }}} T_{j, \mathrm{res}}
\end{aligned}
$$

where $P_{j}\left(P_{j}^{1}\right)$ are the (associated) Legendre-polynomials in $\cos \theta_{\text {c.m. }}$.

The resonant excursion observed in a specifi c $p p$ elastic scattering observable is obtained mainly from the interference term of the resonant and non-resonant amplitudes. Here, we will only discuss unpolarized differential cross-sections and analyzing powers, given by

$$
\begin{aligned}
\frac{\mathrm{d} \sigma}{\mathrm{d} \Omega_{\mathrm{c} . \mathrm{m} .}} & =\frac{1}{k^{2}}\left(\sum_{i=1}^{5}\left|H_{i}\right|^{2}+\left|H_{4}\right|^{2}\right) \\
A_{N} \frac{\mathrm{d} \sigma}{\mathrm{d} \Omega_{\mathrm{c} . \mathrm{m} .}} & =\frac{2}{k^{2}} \operatorname{Im}\left[\left(H_{3}+H_{5}\right) H_{4}^{*}\right],
\end{aligned}
$$

with $k$ being the wave number, i.e. the proton c.m. momentum divided by $\hbar\left(k=p_{\text {c.m. }} / \hbar\right)$. For a resonance in a spin-singlet partial wave, for example, we obtain

$$
\frac{\mathrm{d} \sigma}{\mathrm{d} \Omega_{\mathrm{c} . \mathrm{m} .}}=\left.\frac{\mathrm{d} \sigma}{\mathrm{d} \Omega_{\mathrm{c} . \mathrm{m} .}}\right|_{\mathrm{nr}}-\frac{2}{k^{2}}(2 j+1) P_{j} \operatorname{Re}\left[H_{1, \mathrm{nr}}^{*} T_{j, \mathrm{res}}\right]
$$

where the resonant excursion will have a characteristic dependence on $\theta_{\text {c.m. }}$ through the corresponding Legendre-polynomials. Note, that expected excursions are small, so that we have dropped the term quadratic in $T_{j \text {,res }}$. It is straightforward to obtain similar expressions for other observables. It should be emphasized that the resonance excursion of a single isolated narrow resonance is proportional to the elasticity $\eta_{\mathrm{el}}=\Gamma_{\mathrm{el}} / \Gamma_{\mathrm{tot}}$ (see Eqs. 3 - 7).

\subsection{Experimental Data}

The analysis presented here is based on excitation functions of the unpolarized differential cross-section [42] obtained by the EDDA-experiment at COSY. Of the cited errors only those are taken into account which give rise to point-to-point fluctuations in angular distributions or excitation functions. Other systematic uncertainties are smoothly varying with energy or scattering angle and will not give rise to narrow structures in excitation functions.

In principle, the excitation functions for the analyzing power $[43,44]$ and spin correlation parameter [46] of the same experiment can also be used. However, the analyzing power data give little additional constraints on resonant contribution, with the exception of wider resonances in spin-triplet partial waves. The excitation functions of the spin correlation parameters $A_{N N}$, $A_{S S}$ and $A_{S L}$ [46]. were not used at all because those data have a rather low statistical accuracy when compared to the cross-section data.

Only the excitation functions of the unpolarized differential cross-section [42] are used to test the compatibility with an assumed resonance, and we will call this data the test data set in the following sections.

\subsection{Effective Resolution Function}

EDDA is an internal target experiment that measures the excitation functions quasi continuously during the beam acceleration of the cooler synchrotron COSY. The momentum resolution of the internal proton beam corresponds to a momentum spread of about $0.4 \mathrm{MeV} / \mathrm{c}$ yielding a rms width of about 
$0.13 \mathrm{MeV}$ in the $p p$ c.m.-system. However, the effective resolution is determined by the momentum bin width. The data are recorded during the beam acceleration in time bins of $2.5 \mathrm{~ms}$. The speed of the linear momentum ramp is $1.15 \mathrm{GeV} / \mathrm{c}$. Therefore, the corresponding width of the elementary momentum bin is $2.875 \mathrm{MeV} / \mathrm{c}$. But, in order to increase statistics the data are binned in bins with a width of $\Delta p=25 \mathrm{MeV} / \mathrm{c}$. Thus, the momentum resolution is determined by a momentum width of $25 \mathrm{MeV} / \mathrm{c}$. The corresponding energy width $\Delta W$ of the $p p$ c.m.-system is about $8.5 \mathrm{MeV}(8.3 \mathrm{MeV}$ at $W=2.1 \mathrm{GeV}$, $8.8 \mathrm{MeV}$ at $W=2.4 \mathrm{GeV}$ and $8.2 \mathrm{MeV}$ at $W=2.7 \mathrm{GeV}$ ). This c.m.-energy width can be taken into account by folding the theoretical excitation functions with a rectangular resolution function corresponding to a momentum bin width of $25 \mathrm{MeV} / \mathrm{c}$ before comparing with the data. However, in order to save computer time the effect of folding was taken into account a posteriori by estimating a correction factor for the tests of the narrowest resonances. i.e. for $\Gamma_{\text {tot }}=10 \mathrm{MeV}$ and $\Gamma_{\text {tot }}=20 \mathrm{MeV}$. This correction factor never exceeds 1.2. For larger total widths the effect of folding is negligible.

\subsection{Phase Shift Analysis}

To model the contribution from non-resonant scattering we use a parameterization in terms of phase shifts as provided by the VPI/GWU PSA solution SP00 [66]. In order to achieve an optimal fi $t$ to the unpolarized differential cross-section data of the EDDA experiment at COSY [42], we modifi ed the experimental data base to exclude all unpolarized differential crosssection data of other experiments in the same energy range and refi tted the phase shift parameters. This ensures an optimum parametrization of the EDDA data and thus a low $\chi^{2}$ for the nonresonant hypothesis. That means, all spin dependent data (including spin dependent EDDA at COSY results) remained and only the unpolarized differential cross-section data from other experiments were removed. The norms of the $\mathrm{d} \sigma / \mathrm{d} \Omega$ data of EDDA at COSY were fi xed to 1.0. The spin dependent data were normalized in accordance with the solution SP00 of [66]. The same data base was also used when a resonance was added to a certain partial wave amplitude and the phase-shift parameters were refi tted to obtain the best representation of the data for the resonance hypothesis. The norms of the $\mathrm{d} \sigma / \mathrm{d} \Omega$ data of EDDA at COSY remained the same for the nonresonant PSA as well as the resonant PSA' solution.

\section{$3.6 \chi^{2}$-Test}

The compatibility of the experimental data with a hypothesis is subject to a $\chi^{2}$-test. We only include the data measured within $\pm \Gamma_{\text {tot }}$ around the energy $W_{\mathrm{R}}$ of the resonance. Including more data, e.g. within $\pm 2 \Gamma_{\text {tot }}$ would simply dilute the information, since here the resonant excursion is already small.

The $\chi^{2}$ obtained from the comparison of the hypothesis to the data is subject to a standard $\chi^{2}$-test [67]. Assuming the $\chi^{2}$ is statistically distributed according to a $\chi^{2}$-distribution $f_{n}$ with the appropriate number $n$ of degrees of freedom, we can calculate the confi dence level (CL) with which we can support or falsify the hypothesis. Assuming the hypothesis were true, the

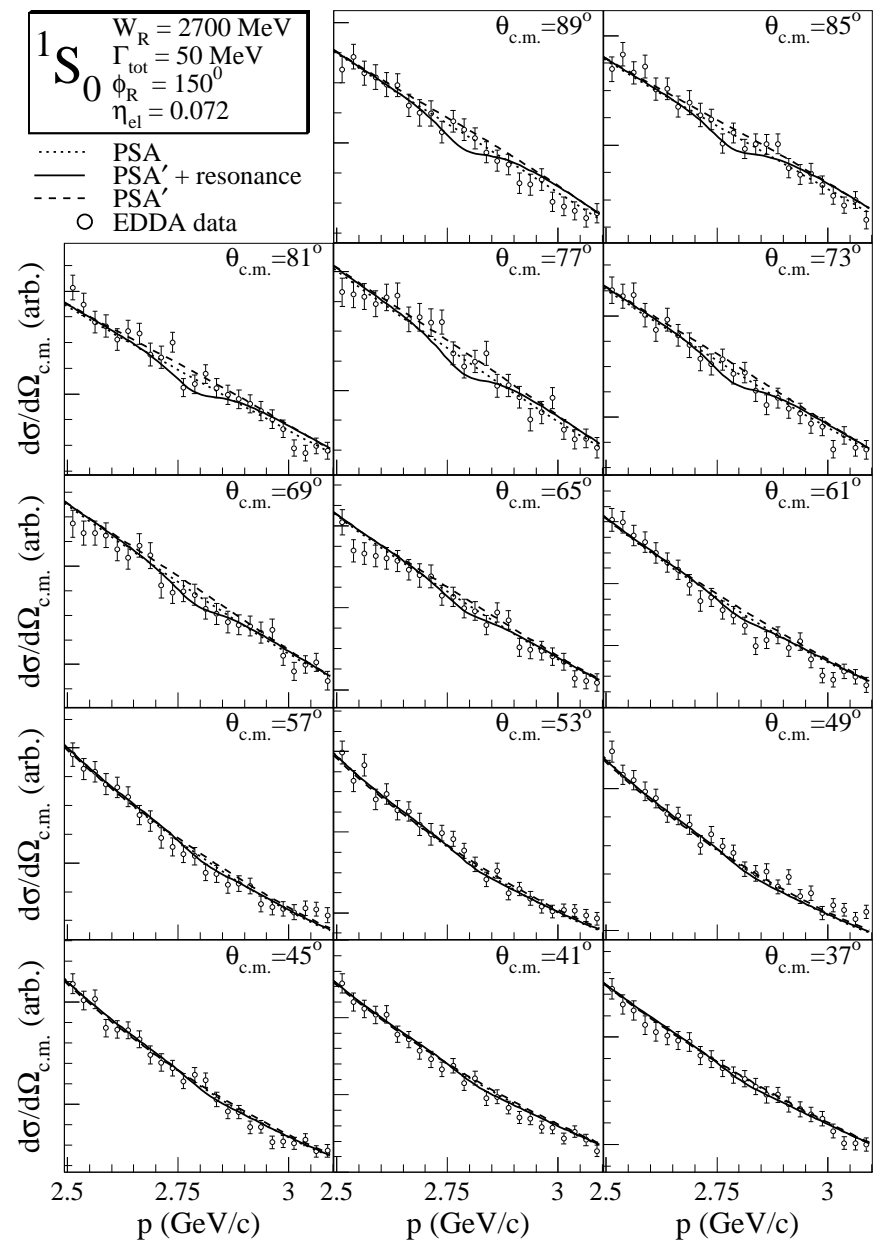

Fig. 3. Excitation functions of $p p$ elastic scattering cross-sections [42] in comparison to a PSA representing the null-hypothesis (dotted line), a PSA best reproducing the world data base when a resonance in the ${ }^{1} S_{0}$ partial wave with parameters as given in the upper left box (resonance position $W_{\mathrm{R}}$, total width $\Gamma_{\text {tot }}$, resonance phase $\phi_{\mathrm{R}}$, elasticity $\eta_{\mathrm{el}}$ ) is added (solid (dashed) line: PSA' with (without) adding the resonance). The scale of $\mathrm{d} \sigma / \mathrm{d} \Omega$ is linear, the zero is suppressed.

probability to obtain a larger $\chi^{2}$ than the observed one in an infi nite repetition of the experiment is given by

$$
P=\int_{\chi^{2}}^{\infty} \mathrm{d} u f_{n}(u) .
$$

If $P$ is very small either the hypothesis is wrong or the current measurement is due to a very unlikely statistical fluctuation. The CL for excluding the hypothesis tested is given by $1-P$, i.e. for a confi dence level of $99 \%$ the probability to observe a larger $\chi^{2}$ when repeating the measurement is only $1 \%$.

We use the number of data points within the c.m. total energy interval $W_{\mathrm{R}} \pm \Gamma_{\text {tot }}$ as the number of degrees of freedom. Thus we neglect the degrees of freedom introduced by the PSA modeling the nonresonant background. This PSA contains roughly 100 parameters, but these are adjusted by using the complete data base of about 20000 data points and cannot be considered as free parameters. 
Table 2. Upper limits (99\% CL) on $\eta_{\mathrm{el}}=\Gamma_{\mathrm{el}} / \Gamma_{\text {tot }}$ based on the differential cross section data of [42].

\begin{tabular}{|c|c|c|c|c|c|c|c|c|c|c|c|c|c|c|c|}
\hline \multirow{4}{*}{$\begin{array}{c}W_{\mathrm{R}} \\
\mathrm{GeV}\end{array}$} & \multicolumn{6}{|c|}{$\overline{\Gamma_{\mathrm{tot}}}=10 \mathrm{MeV}$} & \multicolumn{4}{|c|}{$\overline{\Gamma_{\mathrm{tot}}=20 \mathrm{MeV}}$} & \multicolumn{5}{|c|}{$\overline{\Gamma_{\text {tot }}}=30 \mathrm{MeV}$} \\
\hline & ${ }^{1} S_{0}$ & ${ }^{1} D_{2}$ & ${ }^{3} P_{0}$ & ${ }^{3} P_{1}$ & ${ }^{3} F_{3}$ & ${ }^{1} S_{0}$ & ${ }^{1} D_{2}$ & ${ }^{3} P_{0}$ & ${ }^{3} P_{1}$ & ${ }^{3} F_{3}$ & ${ }^{1} S_{0}$ & ${ }^{1} D_{2}$ & ${ }^{3} P_{0}$ & ${ }^{3} P_{1}$ & ${ }^{3} F_{3}$ \\
\hline & \multicolumn{5}{|c|}{$\eta_{\mathrm{e} l, \max }$} & \multicolumn{5}{|c|}{$\eta_{\mathrm{e} l, \max }$} & \multicolumn{5}{|c|}{$\eta_{\mathrm{el}, \max }$} \\
\hline & $\%$ & $\%$ & $\%$ & $\%$ & $\%$ & $\%$ & $\%$ & $\%$ & $\%$ & $\%$ & $\%$ & $\%$ & $\%$ & $\%$ & $\%$ \\
\hline 2.05 & 7.2 & 3.8 & 9.3 & 2.0 & 3.2 & 7.6 & 2.7 & 9.2 & 1.9 & 2.8 & 7.3 & 2.6 & 8.9 & 1.9 & 2.7 \\
\hline 2.10 & 6.8 & 5.7 & 7.6 & 2.1 & 4.9 & 4.3 & 5.0 & 5.4 & 1.2 & 3.7 & 4.0 & 5.0 & 5.0 & 1.2 & 3.5 \\
\hline 2.15 & 16.2 & 8.5 & 12.7 & 4.8 & 10.4 & 11.4 & 6.3 & 9.4 & 3.1 & 7.3 & 10.8 & 10.0 & 8.5 & 2.9 & 8.3 \\
\hline 2.20 & 5.8 & 6.7 & 9.8 & 1.8 & 7.9 & 5.9 & 4.8 & 7.7 & 1.8 & 6.0 & 6.5 & 5.3 & 8.8 & 2.1 & 7.2 \\
\hline 2.25 & 4.5 & 3.8 & 6.7 & 1.7 & 5.4 & 3.9 & 3.4 & 6.3 & 1.4 & 5.7 & 4.3 & 3.8 & 7.5 & 1.4 & 6.5 \\
\hline 2.30 & 11.5 & 6.0 & 12.1 & 4.2 & 7.8 & 8.6 & 4.6 & 9.2 & 3.2 & 6.3 & 8.3 & 4.3 & 8.9 & 3.1 & 6.2 \\
\hline 2.35 & 6.8 & 4.0 & 7.7 & 2.8 & 6.8 & 5.4 & 2.8 & 5.9 & 2.3 & 5.3 & 6.4 & 3.0 & 6.9 & 2.7 & 5.6 \\
\hline 2.40 & 6.2 & 3.4 & 6.7 & 2.5 & 5.2 & 4.9 & 2.8 & 5.4 & 2.2 & 4.4 & 4.9 & 2.7 & 5.6 & 2.4 & 4.8 \\
\hline 2.45 & 7.0 & 4.3 & 8.6 & 3.3 & 7.7 & 5.6 & 3.2 & 6.1 & 2.6 & 5.8 & 5.1 & 2.9 & 5.6 & 2.6 & 5.3 \\
\hline 2.50 & 7.8 & 4.9 & 8.1 & 2.9 & 9.0 & 5.0 & 3.0 & 5.7 & 2.1 & 6.0 & 4.5 & 2.8 & 5.1 & 1.9 & 5.1 \\
\hline 2.55 & 8.0 & 5.0 & 7.3 & 2.9 & 7.4 & 5.5 & 3.2 & 5.5 & 2.1 & 4.9 & 4.9 & 3.2 & 5.0 & 1.9 & 4.5 \\
\hline 2.60 & 8.3 & 5.5 & 6.9 & 2.9 & 5.6 & 6.0 & 3.7 & 6.2 & 2.2 & 4.6 & 5.8 & 3.6 & 6.2 & 2.1 & 4.3 \\
\hline 2.65 & 7.9 & 4.5 & 8.2 & 3.2 & 3.6 & 6.2 & 3.6 & 6.2 & 2.4 & 2.8 & 5.6 & 3.7 & 6.1 & 2.2 & 2.9 \\
\hline 2.70 & 9.5 & 5.0 & 7.4 & 3.3 & 3.9 & 7.5 & 3.3 & 6.5 & 2.7 & 3.1 & 7.2 & 3.5 & 6.5 & 2.5 & 3.0 \\
\hline 2.75 & 10.5 & 5.0 & 7.3 & 3.6 & 3.2 & 8.8 & 4.8 & 6.4 & 3.0 & 2.8 & 8.5 & 4.7 & 5.6 & 2.9 & 2.8 \\
\hline 2.80 & 25.0 & 10.0 & 9.2 & 5.4 & 3.7 & 15.2 & 6.2 & 7.3 & 3.8 & 2.8 & 13.2 & 5.6 & 6.4 & 3.9 & 2.7 \\
\hline \multirow[t]{3}{*}{2.85} & 11.2 & 5.1 & 8.0 & 5.3 & 3.7 & 12.7 & 6.1 & 9.5 & 6.7 & 4.3 & 11.6 & 6.8 & 12.1 & 9.0 & 5.7 \\
\hline & \multicolumn{6}{|c|}{$\Gamma_{\mathrm{tot}}=40 \mathrm{MeV}$} & \multicolumn{4}{|c|}{$\Gamma_{\mathrm{tot}}=50 \mathrm{MeV}$} & \multicolumn{5}{|c|}{$\Gamma_{\mathrm{tot}}=60 \mathrm{MeV}$} \\
\hline & ${ }^{1} S_{0}$ & ${ }^{1} D_{2}$ & ${ }^{3} P_{0}$ & ${ }^{3} P_{1}$ & ${ }^{3} F_{3}$ & ${ }^{1} S_{0}$ & ${ }^{1} D_{2}$ & ${ }^{3} P_{0}$ & ${ }^{3} P_{1}$ & ${ }^{3} F_{3}$ & ${ }^{1} S_{0}$ & ${ }^{1} D_{2}$ & ${ }^{3} P_{0}$ & ${ }^{3} P_{1}$ & ${ }^{3} F_{3}$ \\
\hline$W_{\mathrm{R}}$ & \multicolumn{5}{|c|}{$\eta_{\mathrm{e} l, \max }$} & & & $\eta_{\mathrm{el}}$ & $\operatorname{nax}$ & & & & $\eta_{\mathrm{el}, m a}$ & & \\
\hline $\mathrm{GeV}$ & $\%$ & $\%$ & $\%$ & $\%$ & $\%$ & $\%$ & $\%$ & $\%$ & $\%$ & $\%$ & $\%$ & $\%$ & $\%$ & $\%$ & $\%$ \\
\hline 2.10 & 4.4 & 5.5 & 5.6 & 1.2 & 3.2 & 4.7 & 5.2 & 6.7 & 1.2 & 3.1 & 4.8 & 4.5 & 7.2 & 1.4 & 2.9 \\
\hline 2.15 & 11.5 & 10.2 & 9.6 & 3.0 & 9.6 & 11.9 & 10.6 & 10.1 & 3.2 & 10.0 & 12.6 & 10.5 & 9.8 & 3.6 & 12.1 \\
\hline 2.20 & 8.2 & 6.2 & 9.5 & 2.4 & 9.1 & 8.9 & 7.3 & 10.8 & 2.9 & 15.4 & 10.4 & 8.2 & 12.5 & 3.5 & 18.2 \\
\hline 2.25 & 5.2 & 4.7 & 9.8 & 1.7 & 8.1 & 5.3 & 5.0 & 10.8 & 1.7 & 9.0 & 5.2 & 5.6 & 11.3 & 1.8 & 10.2 \\
\hline 2.30 & 9.1 & 4.5 & 9.4 & 3.2 & 6.6 & 9.7 & 4.6 & 10.1 & 3.5 & 7.0 & 10.2 & 4.7 & 10.7 & 3.2 & 7.2 \\
\hline 2.35 & 7.5 & 3.3 & 8.3 & 5.4 & 5.9 & 8.6 & 3.5 & 9.3 & 5.7 & 6.3 & 10.5 & 3.9 & 11.1 & 6.5 & 6.8 \\
\hline 2.40 & 5.3 & 2.6 & 5.8 & 2.5 & 4.6 & 5.5 & 2.8 & 6.4 & 2.9 & 4.8 & 6.7 & 3.0 & 14.5 & 6.6 & 5.1 \\
\hline 2.45 & 5.2 & 3.0 & 5.9 & 3.0 & 5.2 & 5.6 & 3.1 & 6.7 & 6.8 & 5.4 & 5.7 & 3.1 & 7.5 & 8.4 & 5.3 \\
\hline 2.50 & 4.3 & 2.9 & 5.0 & 1.9 & 4.9 & 4.6 & 3.1 & 5.5 & 2.1 & 5.0 & 5.1 & 3.7 & 6.3 & 2.7 & 5.3 \\
\hline 2.55 & 4.8 & 3.2 & 5.0 & 1.9 & 4.3 & 5.2 & 3.5 & 5.5 & 2.1 & 4.4 & 5.3 & 3.7 & 5.6 & 2.1 & 4.4 \\
\hline 2.60 & 6.0 & 3.7 & 6.5 & 2.2 & 4.3 & 6.3 & 4.1 & 7.0 & 2.4 & 4.7 & 6.7 & 4.3 & 7.5 & 2.5 & 4.7 \\
\hline 2.65 & 5.7 & 3.9 & 5.9 & 2.2 & 3.0 & 5.8 & 4.7 & 6.0 & 2.2 & 3.1 & 5.8 & 4.7 & 6.2 & 2.3 & 3.1 \\
\hline 2.70 & 7.1 & 3.8 & 6.3 & 2.6 & 3.1 & 7.2 & 3.8 & 6.1 & 2.8 & 3.1 & 7.4 & 4.2 & 6.3 & 2.9 & 3.2 \\
\hline 2.75 & 8.5 & 4.5 & 5.6 & 3.2 & 3.1 & 8.7 & 4.9 & 6.1 & 3.4 & 3.4 & 9.3 & 5.2 & 6.6 & 3.7 & 3.5 \\
\hline 2.80 & 12.8 & 5.4 & 6.3 & 4.3 & 2.7 & 13.6 & 5.5 & 6.8 & 4.7 & 3.0 & 13.9 & 5.7 & 7.6 & 5.5 & 3.3 \\
\hline & & & $\Gamma_{\mathrm{tot}}=$ & $0 \mathrm{MeV}$ & & & & $\Gamma_{\mathrm{tot}}=$ & $0 \mathrm{MeI}$ & & & & $\mathrm{t}=90$ & $\mathrm{IeV}$ & \\
\hline & ${ }^{1} S_{0}$ & ${ }^{1} D_{2}$ & ${ }^{3} P_{0}$ & ${ }^{3} P_{1}$ & ${ }^{3} F_{3}$ & ${ }^{1} S_{0}$ & ${ }^{1} D_{2}$ & ${ }^{3} P_{0}$ & ${ }^{3} P_{1}$ & ${ }^{3} F_{3}$ & ${ }^{1} S_{0}$ & ${ }^{1} D_{2}$ & ${ }^{3} P_{0}$ & ${ }^{3} P_{1}$ & ${ }^{3} F_{3}$ \\
\hline$W_{\mathrm{R}}$ & & & & & & & & $\eta_{\mathrm{el}}$ & & & & & $\eta_{\mathrm{el}, m a}$ & & \\
\hline $\mathrm{GeV}$ & $\%$ & $\%$ & $\%$ & $\%$ & $\%$ & $\%$ & $\%$ & $\%$ & $\%$ & $\%$ & $\%$ & $\%$ & $\%$ & $\%$ & $\%$ \\
\hline 2.15 & 12.7 & 9.8 & 10.6 & 3.7 & 10.0 & 12.6 & 12.6 & 11.0 & 3.7 & 8.8 & & & & & \\
\hline 2.20 & 12.3 & 9.8 & 13.6 & 4.0 & 18.9 & 14.8 & 11.7 & 15.0 & 4.7 & 22.8 & 16.8 & 22.1 & 17.0 & 5.4 & 22.8 \\
\hline 2.25 & 5.3 & 5.9 & 10.5 & 1.9 & 11.0 & 5.4 & 6.4 & 10.7 & 2.2 & 11.8 & 5.9 & 6.8 & 12.3 & 2.4 & 22.0 \\
\hline 2.30 & 11.4 & 5.1 & 11.6 & 3.3 & 8.2 & 13.1 & 5.5 & 12.9 & 3.6 & 10.3 & 14.4 & 5.9 & 14.0 & 3.8 & 9.9 \\
\hline 2.35 & 12.6 & 4.2 & 15.0 & 6.8 & 7.1 & 14.0 & 4.4 & 13.3 & 7.0 & 7.3 & 16.8 & 4.9 & 14.5 & 7.3 & 8.1 \\
\hline 2.40 & 7.5 & 3.1 & 17.0 & 6.8 & 5.4 & 9.4 & 3.3 & 17.2 & 6.9 & 5.9 & 10.6 & 3.8 & 19.4 & 7.5 & 6.6 \\
\hline 2.45 & 6.0 & 3.3 & 16.3 & 8.6 & 5.6 & 7.1 & 3.4 & 16.4 & 8.7 & 5.8 & 11.0 & 4.6 & 16.5 & 8.8 & 6.2 \\
\hline 2.50 & 5.7 & 4.0 & 7.4 & 10.3 & 5.6 & 6.1 & 3.7 & 8.5 & 8.5 & 5.8 & 6.3 & 4.5 & 10.0 & 8.4 & 6.0 \\
\hline 2.55 & 5.5 & 4.0 & 6.3 & 2.2 & 4.7 & 5.9 & 4.3 & 7.0 & 2.4 & 4.9 & 6.3 & 4.6 & 7.7 & 8.1 & 5.2 \\
\hline 2.60 & 6.7 & 5.4 & 8.0 & 2.5 & 4.7 & 6.9 & 6.0 & 8.2 & 2.5 & 4.7 & 7.2 & 6.2 & 8.9 & 2.6 & 4.8 \\
\hline 2.65 & 6.2 & 4.8 & 6.2 & 2.3 & 3.2 & 6.8 & 5.1 & 6.9 & 2.5 & 3.5 & 7.2 & 5.5 & 7.3 & 2.6 & 3.8 \\
\hline 2.70 & 7.6 & 4.6 & 6.4 & 3.0 & 3.6 & 8.3 & 4.8 & 7.0 & 3.4 & 3.9 & 9.5 & 5.0 & 7.2 & 4.0 & 4.4 \\
\hline 2.75 & 10.3 & 5.5 & 7.1 & 4.2 & 3.7 & 11.1 & 6.0 & 7.7 & 4.7 & 4.2 & 12.0 & 6.0 & 8.3 & 5.1 & 4.4 \\
\hline 2.80 & 14.9 & 6.0 & 8.3 & 6.2 & 3.7 & & & & & & & & & & \\
\hline
\end{tabular}




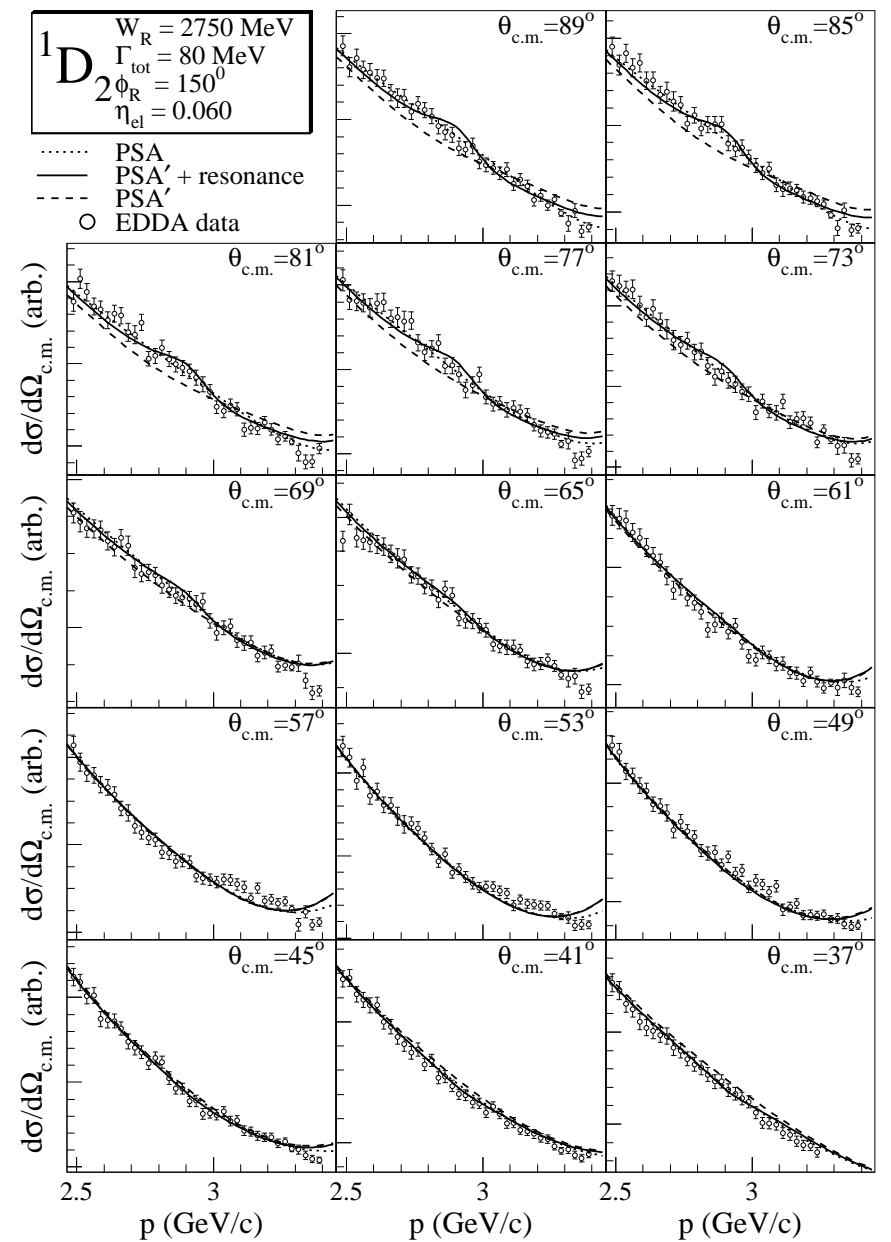

Fig. 4. The same as Fig. 3, but for a resonance in the ${ }^{1} D_{2}$ partial wave.

Table 3. Upper limits (99\% CL) on $\eta_{\mathrm{el}}$ for $\Gamma_{\mathrm{tot}}=100 \mathrm{MeV}$ as in Tab. 2.

\begin{tabular}{rrrrrr}
\hline \hline \multicolumn{5}{c}{$\Gamma_{\text {tot }}=100 \mathrm{MeV}$} \\
& ${ }^{1} S_{0}$ & ${ }^{1} D_{2}$ & ${ }^{3} P_{0}$ & ${ }^{3} P_{1}$ & ${ }^{3} F_{3}$ \\
$W_{\mathrm{R}}$ & \multicolumn{5}{c}{$\eta_{\mathrm{el}, \text { max }}$} \\
$\mathrm{GeV}$ & $\%$ & $\%$ & $\%$ & $\%$ & $\%$ \\
\hline 2.20 & 18.9 & 16.4 & 18.2 & 5.8 & 22.6 \\
2.25 & 6.8 & 7.6 & 12.7 & 2.5 & 17.7 \\
2.30 & 14.0 & 6.2 & 14.9 & 3.5 & 23.8 \\
2.35 & 19.7 & 5.2 & 16.1 & 7.5 & 9.3 \\
2.40 & 13.1 & 6.4 & 20.7 & 7.9 & 7.2 \\
2.45 & 11.5 & 4.9 & 16.7 & 8.9 & 6.4 \\
2.50 & 6.7 & 4.0 & 16.7 & 8.3 & 6.3 \\
2.55 & 6.8 & 5.5 & 8.9 & 10.4 & 5.7 \\
2.60 & 7.2 & 7.3 & 9.2 & 2.7 & 4.8 \\
2.65 & 7.4 & 5.9 & 7.8 & 2.8 & 4.1 \\
2.70 & 10.7 & 5.8 & 8.0 & 5.8 & 5.1 \\
\hline \hline
\end{tabular}

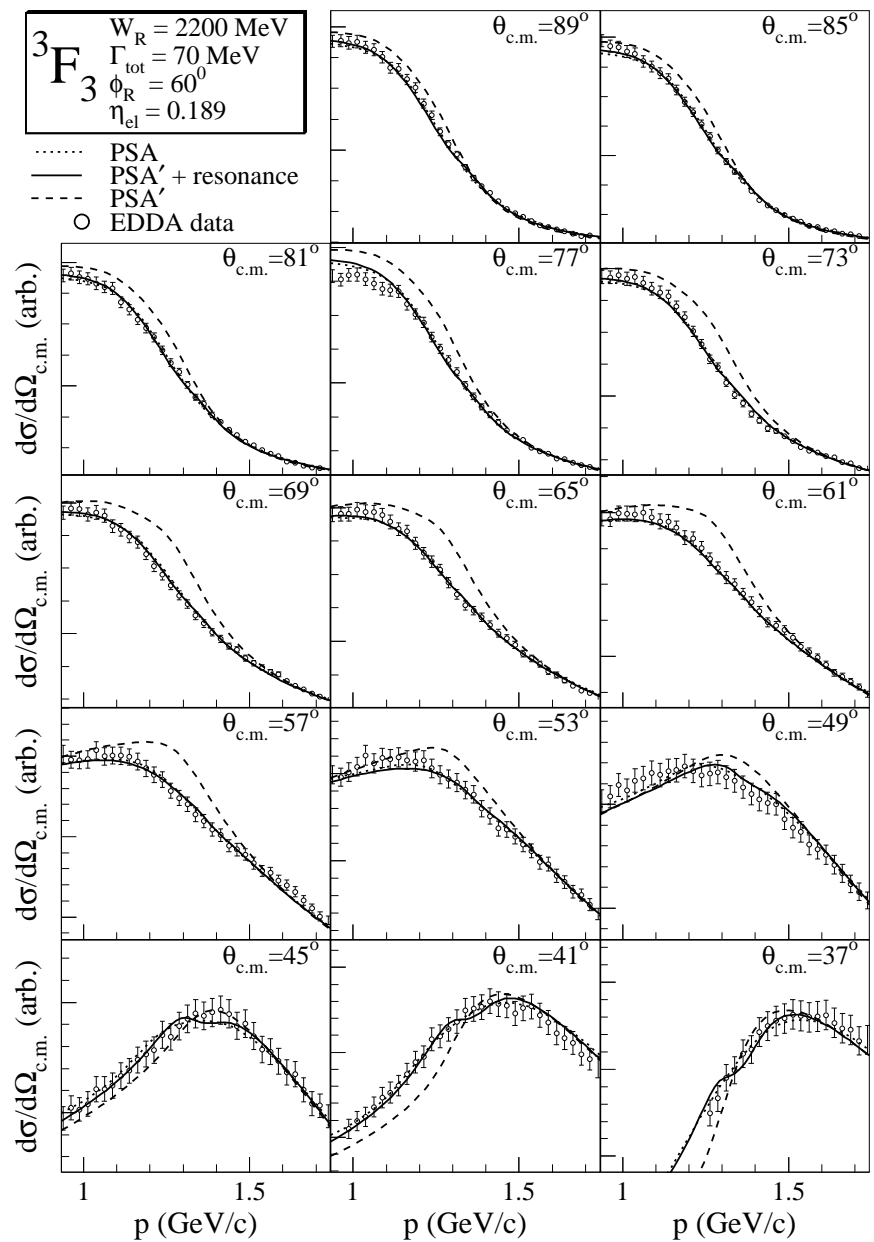

Fig. 5. The same as Fig. 3, but for a resonance in the ${ }^{3} F_{3}$ partial wave.

\section{Results}

The determination of upper limits of resonance contributions was performed for all uncoupled partial waves with $J \leq 3$ and isospin $I=1$, i.e. ${ }^{1} S_{0},{ }^{1} D_{2},{ }^{3} P_{0},{ }^{3} P_{1}$ and ${ }^{3} F_{3}$. These partial waves cover the most important predictions for dibaryonic resonances. Upper limits on the elasticity $\eta_{\mathrm{el}}=\Gamma_{\mathrm{el}} / \Gamma_{\mathrm{tot}}$ are deduced from the differential cross-section data of [42]. For this systematic study the following parameter space was chosen: The resonance energy $W_{\mathrm{R}}$ was varied between 2.05 and $2.85 \mathrm{GeV}$ in $50 \mathrm{MeV}$ steps, the total width $\Gamma_{\text {tot }}$ between 10 and $100 \mathrm{MeV}$ in $10 \mathrm{MeV}$ steps and the relative phase $\phi_{\mathrm{R}}$ between $0^{\circ}$ and $180^{\circ}$ in $10^{\circ}$ steps. The limits in $W_{\mathrm{R}}$ correspond to the energy range of the EDDA-experiment. The lower limit of the total width $\Gamma_{\text {tot }}$ is determined by the fi nite momentum bin width of $25 \mathrm{MeV} / \mathrm{c}$ of the EDDA data corresponding to a c.m. energy width of about $8.5 \mathrm{MeV}$. The upper limit of the total width is a consequence of the applicability of the method.

Typical results are shown in Figs. 3-5 for the analysis of the partial waves ${ }^{1} S_{0},{ }^{1} D_{2}$ and ${ }^{3} F_{3}$. Fig. 6 shows upper limits $(99 \% \mathrm{CL})$ on $\eta_{\mathrm{el}}=\Gamma_{\mathrm{el}} / \Gamma_{\text {tot }}$ for the lowest uncoupled partial waves as a function the resonance energy $W_{\mathrm{R}}$ for three different total widths $\Gamma_{\text {tot }}$. In Tabs. 2 and 3 the upper limits (99\% CL) on $\eta_{\mathrm{el}}=\Gamma_{\mathrm{el}} / \Gamma_{\text {tot }}$ are listed as a function of the resonance energy $W_{\mathrm{R}}$ and the total width $\Gamma_{\text {tot}}$. 

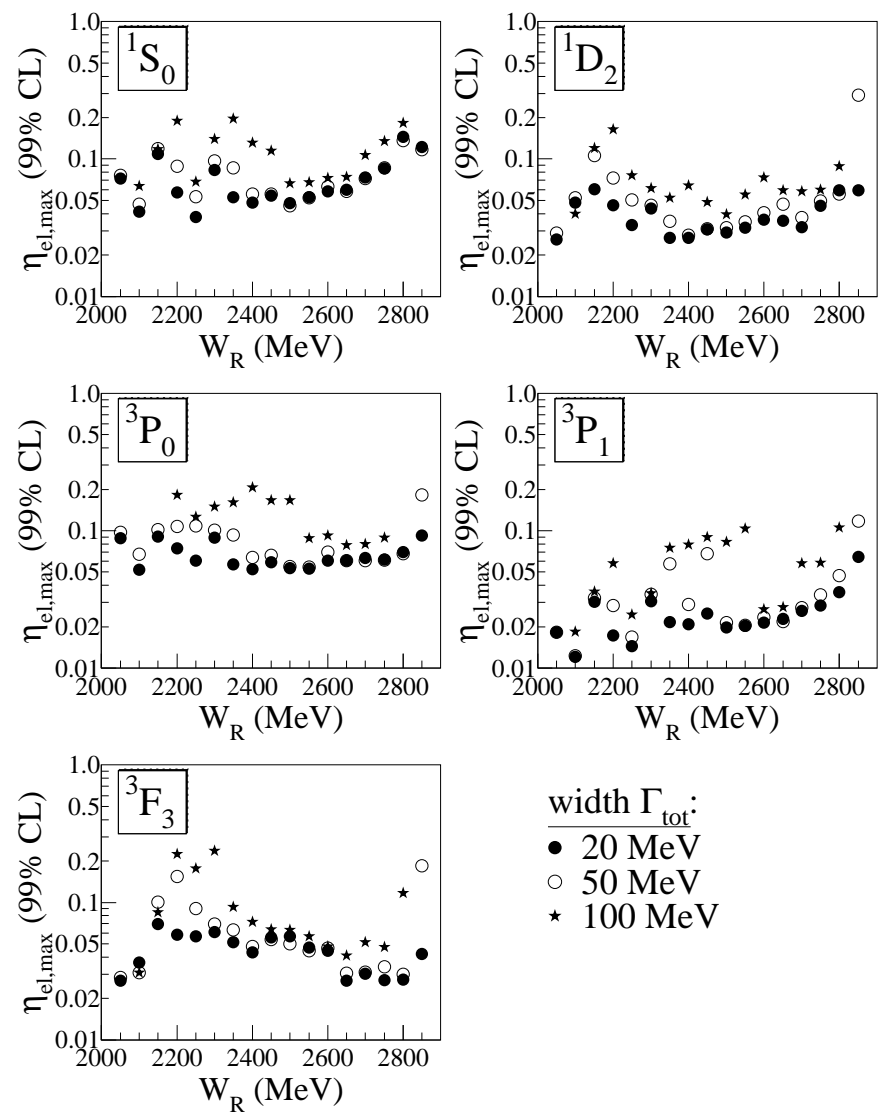

width $\Gamma_{\text {tot }}$ :

- $20 \mathrm{MeV}$

- $50 \mathrm{MeV}$

$\star 100 \mathrm{MeV}$

Fig. 6. Upper limits (99\% CL) on $\eta_{\mathrm{el}}=\Gamma_{\mathrm{el}} / \Gamma_{\text {tot }}$ for the lowest uncoupled partial waves as a function the resonance energy $W_{\mathrm{R}}$ for three different total widths $\Gamma_{\text {tot }}$ based on the cross-section data of [42].

The main results can be summarized as follows:

- Excepting some statistical fluctuations the upper limits of $\eta_{\mathrm{el}}=\Gamma_{\mathrm{el}} / \Gamma_{\text {tot }}$ for narrow resonances $\left(\Gamma_{\mathrm{tot}} \leq 50 \mathrm{MeV}\right)$ show a rather smooth energy dependence. The upper limits are typically about $4-8 \%\left({ }^{1} S_{0}\right), 3-5 \%\left({ }^{1} D_{2}\right), 5-8 \%\left({ }^{3} P_{0}\right)$, 2-3\% ( $\left.{ }^{3} P_{1}\right)$ and 3-6\% $\left({ }^{3} F_{3}\right)$.

- The upper limits of $\eta_{\mathrm{el}}=\Gamma_{\mathrm{el}} / \Gamma_{\mathrm{tot}}$ for $\Gamma_{\mathrm{tot}}>50 \mathrm{MeV}$ are systematically larger.

- For $\Gamma_{\text {tot }}>50 \mathrm{MeV}$ the upper limits of $\eta_{\mathrm{el}}=\Gamma_{\mathrm{el}} / \Gamma_{\text {tot }}$ are relatively large for ${ }^{1} S_{0}$ near 2.20 and $2.35 \mathrm{GeV},{ }^{3} P_{0}$ near 2.20 and $2.40 \mathrm{GeV},{ }^{1} D_{2}$ near $2.15 \mathrm{GeV}$ and ${ }^{3} F_{3}$ near $2.25 \mathrm{GeV}$.

\section{Discussion}

Though many predictions of exotic dibaryon resonances exist with specifi cation of the resonance energy $W_{\mathrm{R}}$ only few paper predict total and partial widths as well. Notable exceptions are studies of isospin $\mathrm{I}=0$ and $\mathrm{I}=1$ dibaryons by Lomon and coworkers [14-16] and studies of dibaryons in the strangeness sector $\mathrm{S}=-1$ by Aerts and Dover [5, 6]. Lomon and coworkers predict the lowest $\mathrm{I}=1,{ }^{1} S_{0}$ dibaryon at $W_{\mathrm{R}}=2.71 \mathrm{GeV}$ with $\Gamma_{\text {tot }}=50 \mathrm{MeV}, \Gamma_{\mathrm{el}}=5 \mathrm{MeV}$ and $\eta_{\mathrm{el}}=10 \%$. Such a resonance is not probable, the upper limit of elasticity (99\% CL) for a ${ }^{1} S_{0}$ resonance at $W_{\mathrm{R}}=2.71 \mathrm{GeV}$ is $\eta_{\mathrm{el}}=7.2 \%$ (see
Tab. 2). The predicted lowest ${ }^{1} D_{2}$ dibaryon at $W_{\mathrm{R}}=2.88 \mathrm{GeV}$ with $\Gamma_{\text {tot }}=100 \mathrm{MeV}, \Gamma_{\mathrm{el}}=5 \mathrm{MeV}$ and $\eta_{\mathrm{el}}=5 \%$ is beyond the range of the present study.

It is interesting to note that in the energy regions of the structures observed in spin-dependent total cross-sections of $p p$ elastic scattering at $2170 \mathrm{MeV}$ and $2250 \mathrm{MeV}$ [47, 48] the upper limits of $\eta_{\mathrm{el}}=\Gamma_{\mathrm{el}} / \Gamma_{\text {tot }}$ are relatively large for ${ }^{1} D_{2}$ and ${ }^{3} F_{3}$ and $\Gamma_{\text {tot }}>50 \mathrm{MeV}$. (see sect. 2).

It should be mentioned that our results are compatible with a previous study of excitation functions of $p p$ elastic scattering analyzing power in the lab energy range 655 - $1017 \mathrm{MeV}$ [28]. In this paper upper limits of the elasticity $\eta_{\mathrm{el}}$ were deduced for two resonances, ${ }^{3} P_{1}, W_{\mathrm{R}}=2192 \mathrm{MeV}, \Gamma_{\text {tot }}=25 \mathrm{MeV}$ and ${ }^{3} F_{3}, W_{\mathrm{R}}=2240 \mathrm{MeV}, \Gamma_{\text {tot }}=16 \mathrm{MeV}$, to be $2 \%$ and $11 \%$, respectively. In [28] it was also noted that the differential crosssection data (of the type that SATURNE [27] and EDDA [41, 42] obtained) provide a more signifi cant constraint for such an analysis than the analyzing power data.

A previous internal target experiment at $\operatorname{KEK}[29,30]$ observed two narrow structures in the excitation function of the analyzing power $A_{N}$ at a kinetic energy near $632 \mathrm{MeV}$ corresponding to $\sqrt{s}=2.17 \mathrm{GeV}$. The authors attempted to interpret the observed anomaly by the effect of a narrow resonance. But the anomalies were not seen in a SATURNE experiment by Beurtey et al. [32]. Also the EDDA experiment [44] did not confi rm the results of the KEK experiment.

In a systematic search for narrow resonances one should also include all measured spin observables. Especially, the spin correlation parameters $A_{N N}$ and $A_{S S}$ exhibit larger sensitivities to a narrow resonance than the unpolarized differential cross-section. However, these observables have not been measured with suffi cient accuracy. Therefore, we omit the inclusion of the spin correlation parameters. In this context, we mention an experiment [33] where a narrow anomaly has been observed in the excitation function of $A_{N N}$ around $2.11 \mathrm{GeV}$ kinetic energy at $\Theta_{\text {c.m. }}=90^{\circ}$. However, this result was not confi rmed in later remeasurements [35].

The upper limits on $\eta_{\mathrm{el}}$ of the present study do not exclude completely the possible existence of exotic narrow resonances in the $p p$ system. Narrow resonances with elasticities below the upper limits given in Tabs. 2 and 3 are still possible.

\section{Summary}

Upper limits of resonance contributions to proton-proton elastic scattering in the c.m. mass range $2.05-2.85 \mathrm{GeV} / \mathrm{c}^{2}$ have been deduced on the basis of recent high-precision data of the internal target experiment EDDA at COSY [42]. In order to test the resonance hypothesis a Breit-Wigner term describing single isolated narrow resonances is introduced into the S-matrix element. The analysis provides an unbiased statistical test on the compatibility of resonance contributions with the measured excitation functions of differential cross-sections. We use a $\chi^{2}$ test in order to deduce upper limits on the elasticity $\eta_{\mathrm{el}}=$ $\Gamma_{\text {el }} / \Gamma_{\text {tot }}$ of resonances in the partial waves ${ }^{1} S_{0},{ }^{1} D_{2},{ }^{3} P_{0},{ }^{3} P_{1}$, and ${ }^{3} F_{3}$ with a $99 \%$ confi dence level.

For narrow resonances $\left(\Gamma_{\text {tot }} \leq 50 \mathrm{MeV}\right)$ the upper limits of $\eta_{\mathrm{el}}=\Gamma_{\mathrm{el}} / \Gamma_{\mathrm{tot}}$ are typically about $4-8 \%\left({ }^{1} S_{0}\right), 3-5 \%\left({ }^{1} D_{2}\right)$, 5-8 \% ( $\left.{ }^{3} P_{0}\right), 2-3 \%\left({ }^{3} P_{1}\right)$ and 3-6 \% $\left({ }^{3} F_{3}\right)$. For resonances 
with $50 \mathrm{MeV}<\Gamma_{\text {tot }}<100 \mathrm{MeV}$ the upper limits of $\eta_{\mathrm{el}}$ are systematically larger.

It is interesting to note that many dibaryonic states have been predicted in the mass range $2.05-2.85 \mathrm{GeV}$. Resonances with total widths below $100 \mathrm{MeV}$ would be good candidates for exotic dibaryonic states. But narrow resonances have not been observed. This might be due to the fact that such narrow states do not exist at all. Another possibility is that the elasticities of such resonances are very small, i.e. below the upper limits listed in Tabs. 2 and 3. In this context it should be mentioned that the ${ }^{1} S_{0}$ dibaryon resonance predicted by Lomon et al. [15] at $W_{\mathrm{R}}=2.71 \mathrm{GeV}$ with $\Gamma_{\text {tot }}=50 \mathrm{MeV}, \Gamma_{\mathrm{el}}=5 \mathrm{MeV}$ and $\eta_{\mathrm{el}}=10 \%$ is not probable. Summarizing, exotic narrow resonances in the $p p$ system with elasticities above the upper limits given in Tabs. 2 and 3 are excluded by the present study.

\section{Acknowledgements}

We are indebted and thankful to Prof. R. A. Arndt for the permission granted to use the software of the VPI/GWU phaseshift analysis, the implementation of additional resonances in the program and his advice in using the code. We also acknowledge helpful discussions with Prof. K. W. Mc Voy. This work recieved fi nancial support from the BMBF (06BN664I(6) and 06HH152) and from FFE, FZ Jülich.

\section{References}

1. R. Jaffe, Phys. Rev. Lett. 38, 195 (1977).

2. P. J. G. Mulders, A. T. M. Aerts, and J. J. de Swart, Phys. Rev. Lett. 40, 1543 (1978).

3. A. T. M. Aerts, P. J. G. Mulders, and J. J. de Swart, Phys. Rev. D17, 260 (1978).

4. P. J. Mulders, A. T. Aerts, and J. J. D. Swart, Phys. Rev. D21, 2653 (1980).

5. A. T. M. Aerts and C. B. Dover, Phys. Lett. 146B, 95 (1984).

6. A. T. M. Aerts and C. B. Dover, Nucl. Phys. B253, 116 (1985).

7. C. W. Wong, Prog. Nucl. Part. Phys. 8, 223 (1982).

8. M. Oka, K. Shimizu, and K. Yazaki, Nucl. Phys. A464, 700 (1987).

9. N. Konno, H. Nakamura, and H. Noya, Phys. Rev. D35, 239 (1987)

10. A. P. Balachandran, A. Barducci, F. Lizzi, V. G. J. Rodgers, and A. Stern, Phys. Rev. Lett. 52, 887 (1984).

11. A. P. Balachandran, F. Lizzi, V. G. J. Rodgers, and A. Stern, Nucl. Phys. B256, 525 (1985).

12. R. L. Jaffe and F. E. Low, Phys. Rev. D19, 2105 (1979).

13. B. L. G. Bakker and P. J. Mulders, Adv. Nucl. Phys. 17, 1 (1986).

14. P. LaFrance and E. L. Lomon, Phys. Rev. D34, 1341 (1986).

15. P. Gonzalez and E. L. Lomon, Phys. Rev. D34, 1351 (1986).

16. P. Gonzalez, P. LaFrance, and E. L. Lomon, Phys. Rev. D35, 2142 (1987).
17. E. L. Lomon and H. Feshbach, Ann. Phys. 48, 94 (1968).

18. E. L. Lomon, Phys. Rev. D26, 576 (1982).

19. E. L. Lomon nucl-th/9710006. (1997).

20. K. Hidaka and A. Yokosawa, Surveys High Energy Phys. 1, 141 (1980).

21. P. Kroll, in Topical Seminar on Few and Many Quark Systems, San Miniato, Italy, 1985, edited by F. Navarria (Istituto Naz. Fis. Nucl., 1985), p. 355.

22. A. Yokosawa, in San Miniato Topical Seminar, San Miniato, Italy, Mar 1985, edited by F. Navarria (Istituto Naz. Fis. Nucl., 1985), p. 383.

23. M. P. Locher, M. E. Sainio, and A. Svarc, Adv. Nucl. Phys. 17, 47 (1986).

24. K. K. Seth, in Proc. Electron and Photon Interactions At Intermediate Energies, edited by D. Menze (Springer Verlag Berlin) Lecture Notes in Physics 234, 150 (1985).

25. K. K. Seth, in Proc. "Baryon-Baryon Interaction and Dibaryonic Systems", Bad Honnef (1988), edited by M.G. Huber (Kernforschungsanlage Jülich) Spezielle Berichte der Kernforschungsanlage Jülich Jül-Spez-501, 12 (1989).

26. A. Yokosawa (Fermilab E-704), Int. Journ. of Mod. Phys. A, Singapore 5, 3089 (1990), presented at 25th Int. Conf. on High-Energy Physics, Singapore, Aug 2-8, 1990.

27. M. Garçon et al., Nucl. Phys. A 445, 669 (1985).

28. M. Garçon et al., Phys. Lett. B183, 273 (1987).

29. H. Shimizu et al., Phys. Rev. C 42, 483 (1990).

30. Y. Kobayashi et al., Nucl. Phys. A569, 791 (1994).

31. J. Nagata, M. Matsuda, N. Hiroshige, and T. Ueda, Phys. Rev. C45, 1432 (1992).

32. R. Beurtey et al., Phys. Lett. B293, 27 (1992).

33. J. Ball et al., Phys. Lett. B 320, 206 (1994).

34. F. Lehar, in Proceedings of the 8th International Conference on the Structure of Baryons, Baryons '98, Bonn, Germany, edited by D. W. Menze and B. Metsch (World Scientifi c, Singapore, 1999), p. 622.

35. J. Ball et al. (NNSaturne II), Proton-proton Data Measured by the Nucleon-nucleon Collaboration at Saturne II, CTU Reports 4, 3 (2000).

36. R. Bilger et al. (LEPS and WASA/PROMICE), Prog. Part. Nucl. Phys. 36, 369 (1996).

37. R. Bilger, Habilitationsschrift, Universität Tübingen (1999).

38. A. S. Khrykin et al., $\pi$ N-Newsletter 10, 67 (1995).

39. A. S. Khrikin, in Proc. $14^{\text {th }}$ Intl. Conf. on Particles and Nuclei, Williamsburg, May 1996, edited by C. E. Carlson and J. J. Domingo (World Scientifi c, Singapore, 1997), p. 533.

40. B. Tatischeff et al., Phys. Rev. C59, 1878 (1999).

41. D. Albers et al. (EDDA), Phys. Rev. Lett. 78, 1652 (1997).

42. D. Albers et al. (EDDA), Eur. Phys. J. A22, 125 (2004).

43. M. Altmeier et al. (EDDA), Phys. Rev. Lett. 85, 1819 (2000).

44. M. Altmeier et al. (EDDA), Eur. Phys. J. A23, 351 (2005).

45. F. Bauer et al. (EDDA), Phys. Rev. Lett. 90, 142301 (2003).

46. F. Bauer et al. (EDDA), Phys. Rev. C71, 054002 (2005).

47. I. P. Auer et al., Phys. Rev. Lett. 41, 354 (1978).

48. E. K. Biegert et al., Phys. Lett. 73B, 235 (1978). 
49. K. Hidaka et al., Phys. Lett. B70, 479 (1977).

50. N. Hoshizaki, Prog. Theor. Phys. 60, 1796 (1978).

51. N. Hoshizaki, Prog. Theor. Phys. 61, 129 (1979).

52. R. Bhandari, R. A. Arndt, L. D. Roper, and B. J. VerWest, Phys. Rev. Lett. 46, 1111 (1981).

53. R. A. Arndt et al., Phys. Rev. D28, 97 (1983).

54. D. V. Bugg, Comments Nucl. Part. Phys. 13, 287 (1984).

55. C. Elster, K. Holinde, D. Schütte, and R. Machleidt, Phys. Rev. C38, 1828 (1988).

56. P. LaFrance, E. L. Lomon, and M. Aw (1993), nucl-th/9306026.

57. T. Ueda, Phys. Lett. B119, 281 (1982).

58. H. Rohdjess, Habilitationsschrift, Universität Bonn (2000).

59. K. O. Eyser, R. Machleidt, and W. Scobel (EDDA), Eur. Phys. J. A22, 105 (2004).

60. R. A. Arndt, C. H. Oh, I. I. Strakovsky, R. L. Workman, and F. Dohrmann, Phys. Rev. C56, 3005 (1997).

61. L. Wolfenstein, Phys. Rev. 85, 1654 (1954).

62. H. P. Stapp, T. J. Ypsilantis, and N. Metropolis, Phys. Rev. 105, 302 (1957).

63. P. Kroll, in Physics Data, edited by H. Behrens and G. Ebel (Fachinformationszentrum Karlsruhe, UniversitätGesamthochschule Wuppertal, 1981), Vol. 22-1.

64. C. Lechanoine-LeLuc and F. Lehar, Rev. Mod. Phys. 65, 47 (1993)

65. K. W. McVoy, in Fundamentals in Nuclear Theory, edited by A. de Shalit and C. Villi (IAEA, Wien, 1967), p. 419.

66. R. A. Arndt, I. I. Strakovsky, and R. L. Workman, Phys. Rev. C 62, 34005 (2000).

67. V. Blobel and E. Lohrmann, Statistische und Numerische Methoden der Datenanalyse (Teubner, Leipzig, 1998). 